\title{
Building quality into contracting and purchasing
}

\author{
Liam Donaldson
}

In 1994 the NHS is still in the early phase of implementation of reforms ${ }^{1}$ which were introduced after a fundamental review of the organisation and funding of health care in Britain. In this new system, in which health care for the population is secured through organisations purchasing services from a range of hospitals and other providers within an internal market, there are three major potential forces which influence quality. ${ }^{2}$

Firstly, there is the tendency for hospitals to compete based on quality: developing new ideas and offering new services or improved ways of delivering existing services should give a competitive edge, the opportunity to win more contracts, and therefore a powerful incentive in terms of income for the hospital. Secondly, there is the tendency for purchasers of service to place contracts based on experience of good and bad care. Thirdly, there is what is specified in the contract itself: the opportunity for purchasers to make explicit, publicly in their contracts, requirements for the quality of service to be provided is a further stimulus for improving quality.

The scope for the purchasing function, through contracts and other means, to improve quality of health care is now perceived as very great ${ }^{3}$ and is seen as yielding the opportunity to move away from the provider dominance which has characterised health care in Britain since the inception of the NHS. Yet in this new NHS environment how far are health authorities and fundholding practices genuinely emerging as the champions of the health needs of local populations?

\section{Problems that must be solved}

In seeking to introduce a new quality impetus within the NHS it is important to recognise that many of the problems which must be addressed are long standing.

Despite a steady downward trend since the beginning of the 1990s waiting times for hospital treatment remain too long and are the aspect of quality which is highest on patients' list of concerns. Variations in access to services, particularly those (such as coronary artery revascularisation procedures) provided in a limited number of hospitals, continue to prevail in a way which does not appear to be related to need. ${ }^{4}$ Doctors still differ greatly in the nature and timing of their interventions in similar clinical situations, ${ }^{5}$ while patients with some conditions (for example, hypertension) remain untreated or inadequately treated when the potential consequences (stroke, coronary heart disease) are very serious. ${ }^{6}$ Moreover, the culture of the health service is not yet one in which complaints and criticisms are welcomed as an opportunity to learn about failures in the quality of care and to make improvements. ${ }^{7}$

The factors which affect the quality of health care delivered to the population of Britain are many and diverse but include the exercise of judgement and skills by individual doctors and other health care professionals; the level, pattern, and distribution of health care facilities within local populations; and purchasing strategies and the setting of contracts by health authorities and fundholding practices. It is the last of these factors which is not only an integral part of the new NHS but the one on which the success of the reforms will largely depend. To the man or woman in the street, organisational changes, even of the magnitude that the British health service has undertaken are not of direct interest. The extent to which health services (however they are organised) deal with problems of the kind described above is the yardstick by which the public will decide whether it supports the health care system of the day. Indeed, the most effective purchaser of health care will not be the one with the most specific or the widest range of quality clauses within contracts but is more likely to be assessed by population based quality measures such as access, equity, and improved outcomes of care.

\section{Purchasing: a quality process}

The concept of purchasing within the NHS is still ill defined. To some, it means no more or less than the exchange of money for services between health authorities and fundholding practices on the one side and hospitals and other service providers on the other. However, in the policy making and management arena purchasing is now a holy grail which is seen as the only true path to health and quality improvement for the future. ${ }^{3}$

The juxtaposition of "purchasing" and "quality" in relation to this debate about achieving change in the health service provokes two main trains of thought. Firstly, purchasing is only one of several mechanisms through which quality of care can be improved. Other mechanisms include wider influences such as professional development and training, empowering consumers, en- 


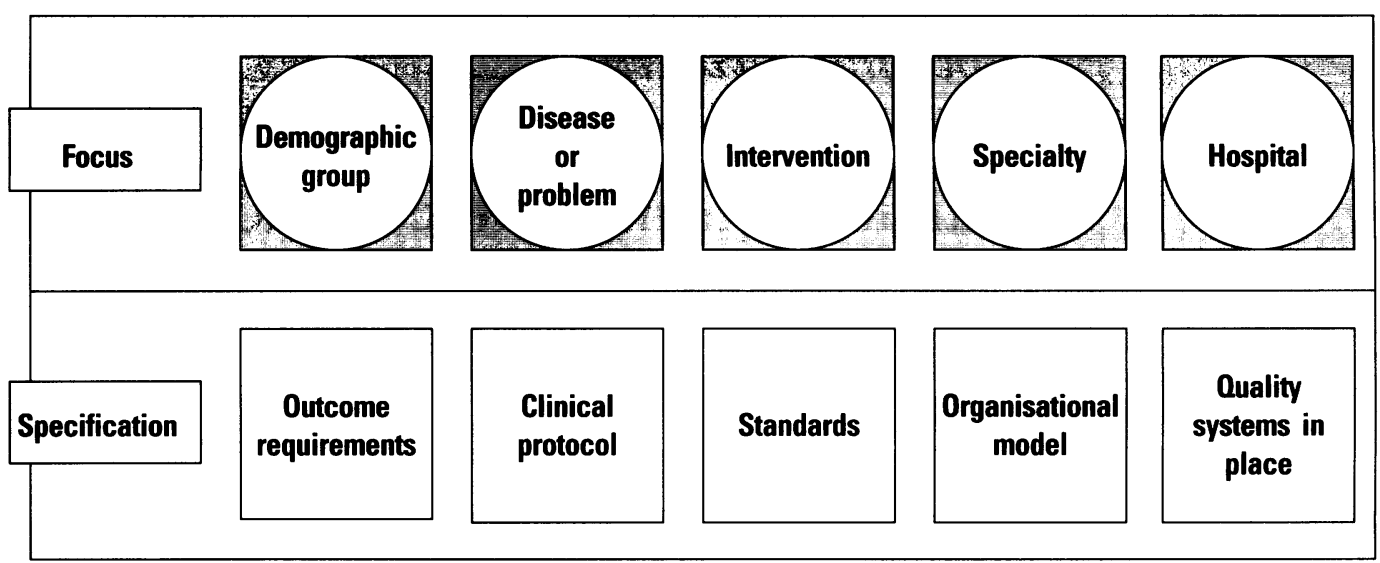

Figure 1 Achieving change in quality through contracting

hancing managerial skills, and using quality improvement methods (such as total quality management and medical audit) at the level of service providers. Secondly, purchasing may be regarded as a process which should be improved.

QUALITY CLAUSES IN CONTRACTS

A particular component of the "purchasing as a lever" approach is the use of quality clauses within contracts as a way to raise standards and achieve improved outcomes of care. Such clauses will differ, depending on the type of contract negotiated between purchaser and provider. Thus if a purchaser sets a contract on a cost per case basis there is more scope to include quite specific statements regarding the quality of medical care than is possible within a block contract. There is a legitimate debate, regardless of the type of contract, as to how far it is appropriate for a purchaser to include specific quality clauses. One central element of the NHS reforms was the recognition of the need for devolution of operational issues to hospital or practice level. The contract must not, therefore, become a mechanism whereby the purchasing organisation can begin to specify detailed procedures to the point where it becomes an operational manager through a different mechanism. At the same time, if health authorities and fundholding practices are to develop a legitimate concern with improving quality of services for their resident or patient populations they will need to be able to go beyond very broad, and effectively meaningless, quality clauses.

The way in which quality clauses are specified within contracts might vary considerably, as seen from the framework of contracting in the matrix shown in figure 1 . The organisational level of a contract could be in terms of demographic or social group (for example, elderly subjects) or disease category (for example, patients who have had acute myocardial infarction, diabetic people); it may be intervention specific (for example, inguinal

\section{Establish requirements}

- Epidemiological assessments

- Consumer views

- Benchmarks of performance

- Implementing technological advances

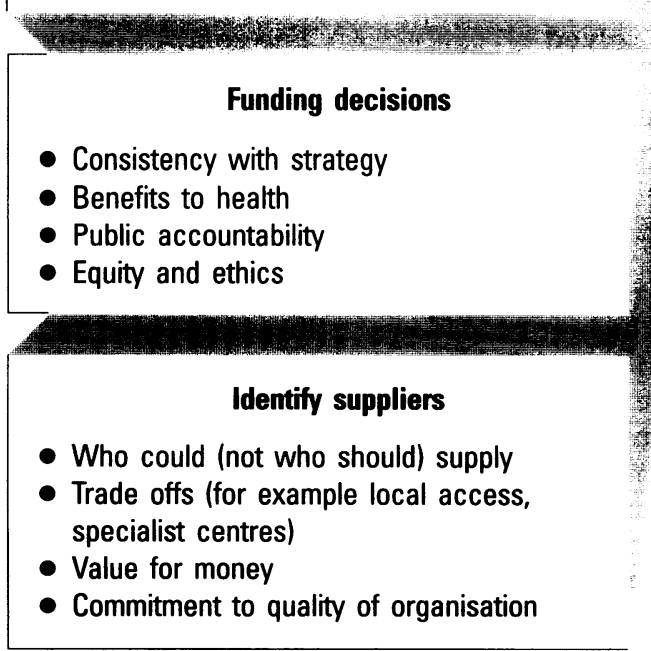

\section{Agree specification}

- Quality criteria

- Common quality values

- Consumer involvement

- Commitment of professional staff

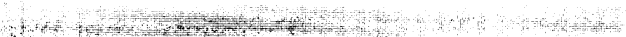

Place contracts

- Choice of supplier

- Detailed negotiation

- Format of contract

- Establish flow of information

\section{Quality management}

- Leadership

- Partnership with supplier

- Evaluate performance

- Further improve the process 
hernia repair, hip joint replacement), specialty specific (for example, dermatology, urology), or on a hospitalwide basis.

The type of quality specification applied to any of these organisational levels in itself could vary in several ways, perhaps along a range of control. At one end of the range the specification might simply be aimed at ensuring that satisfactory medical audit or quality management systems are in place. Though contracts may stimulate quality improvement through their ability to highlight issues, set standards, and require assurance of quality from providers, the whole thrust of modern quality management in other sectors suggests that the best opportunity for improvement will be through developing appropriate and effective systems of quality management at hospital or practice level. This contracting philosophy would contrast (at the other end of the range) with an outcome based approach to specification (fig 1). In this, for an intervention (such as a particular surgical procedure) the purchaser's quality requirements might be expressed in terms of factors such as low complication or recurrence rates.

\section{DEFINING AND IMPROVING THE PROCESS OF} PURCHASING

The second and more fundamental view of quality and purchasing is to view purchasing as a process which should itself be subjected to improvement. This is, of course, a classic total quality management approach. ${ }^{8}$

The steps in the process of purchasing can be set out relatively simply (fig 2 ), but to make the process work effectively it is essential to look in detail at the activities involved in each stage. Figure 3 shows some examples of the main activities, though it is important that those undertaking the process of purchasing should themselves explore issues and identify the ingredients of good quality.

High quality management of the first step of the purchasing process would entail ensuring that the specification of the health care required by the population was based on sound epidemiological assessment of need,

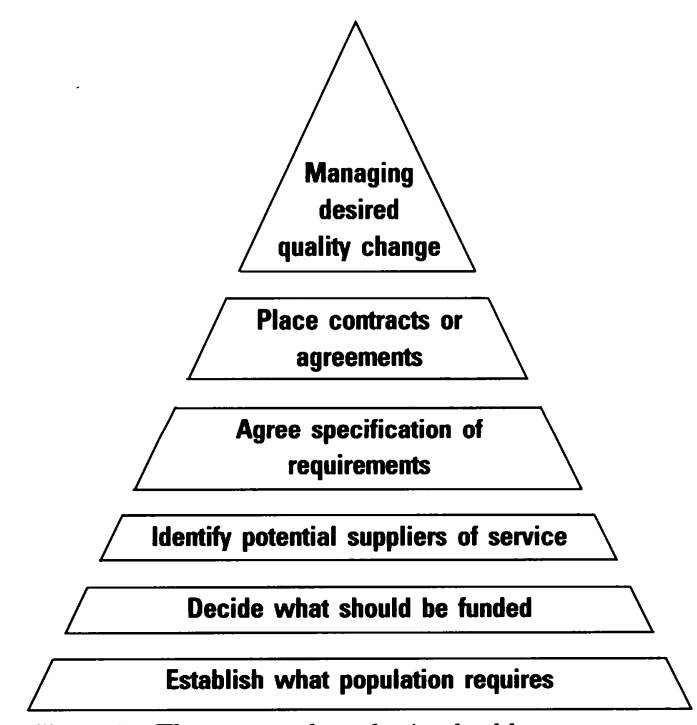

Figure 3 The process of purchasing health care that it involved consumer perspectives, that it took account of the latest advances in medical knowledge and technology, and that comparisons were made with other services known to be doing better.

In the second step a high quality approach would entail ensuring consistency, fairness, and effectiveness in deciding how precisely public funds should be spent. The process of prioritisation is much talked about but there are few examples of it being addressed explicitly in mainstream purchasing. It is at this stage that issues of rationing whether along the lines of Oregon ${ }^{9}$ or otherwise must be debated by the purchasing organisation concerned.

The process of identifying potential suppliers should not be restricted to suppliers who already deliver a particular service. Here is the opportunity for a high quality purchasing function to search for new models of delivery which could offer better care for patients. Good purchasing in this step of the process would also entail being explicit about trade offs - for example, deciding whether local access was more important than the better clinical outcome which might be secured from a more distant specialist unit. Equally, a good purchaser should at this stage seek to ensure that the organisation being contracted with will give value for money and has itself a commitment to quality improvement.

The steps of agreeing the specification of service and actually placing the contract with the chosen hospital or other provider of service requires a more detailed specification of quality requirements. It is here that good purchasing should again seek to take account of consumers' views. Moreover, there are too many anecdotes which suggest that health service managers are agreeing to contracts without taking their medical staff along with them. It is essential that the process of purchasing involves and gains the commitment of the senior professional staff who will actually be delivering the service. Indeed, these phases of the purchasing process essentially concern alignment of the purchasing and supplying organisations. For the process to yield high quality the two types of organisation must develop similar values and share a quality ethos. The approach to information about quality must be open and based on sharing, not concealing, problems.

The success of this whole purchasing process depends on high quality leadership in which all staff are clear about the strategy which must be implemented in a consistent manner. Good quality leadership of the purchasing process will also involve promoting the organisation's goals and philosophy to the local community and all other groups which have the potential to contribute to improvements in health and quality of care. At the present stage of evolution of the health service's internal market there are too many reports of an adversarial relationship existing between health authorities and hospitals. This has echoes of the traditional contracting 
culture of sections of the old NHS, in which competitive tendering was the predominant model. Experience of purchasing in the commercial sector has led to the preferred model in some successful companies being one of a long term relationship based on clear, mutually agreed objectives between purchaser and supplier. This approach, called "partnership sourcing," "could be extended to the NHS, but it is important to recognise that it is not a throwback to the days before the reforms of 1990, when district health authorities managed hospitals in adddition to having responsibilities for the population, when there was no incentive to seek alternative suppliers. Partnership sourcing is not an easy or a soft option in purchasing, it is a tough and uncompromising quest for higher quality in which purchaser and supplier constantly find ways together of doing things better. Finally, a good purchaser of health care should be seeking ways of improving its own process of purchasing.

\section{Conclusion}

The purchasing function created by the 1990 NHS reforms offers a new and major opportunity for quality improvement, but it has yet to be properly conceptualised and used to realise this potential. A commitment by all health care organisations, whether they are providing or purchasing health care, to developing the process of purchasing within a modern quality improvement framework has the potential to produce major gains in the quality of service provided for populations and patients.

1 NHS and Community Care Act 1990, London: HMSO 2 Donaldson RJ, Donaldson LJ. livential public hialth medicinc. Lancaster: Kluwer Academic Press. midillle: 1 ian

3 XHS Management Executive. Parchasing for houlth: frameark for action. London: NHS.ME, I 1903.

t 1)onaldson I.J. Maintaining excellence: the preservation and derclopment of specialised services. In: Smith J, ed. Lomdent after Tomlinson: renganising bis aty madicin. London: BMJ Publishing Group, 1993.

5 I.owry RJS, Donaldson LJ, Gregg PJ. Variations in clinical decision: a study of orthopaedic patients. Putblic Hialt 1991:105:351-5.

6) Smith WCS, Lee AJ, Crombie IK, Tunstall-Pedoe H. Control of blood pressure in Scotland: the rule of halves. B.17f 1990;300:981

7 Donaldson LJ, Cavanagh J. Clinical complaints and thei handling: a time for change. Quality in Heculth Cian 1992;1:21-5.

8 1)eming WE. Out of the irisis. Cambridge: Cambridge Lniversity Press, 1986

c) Crawshaw R. Oregon sets priorities in health care. Bulletw of . Mcidical Ethics 1991;69:32 5.

10) Making parthership sontring happen. I onden: Partnership Sourcing I imited, 1993. 